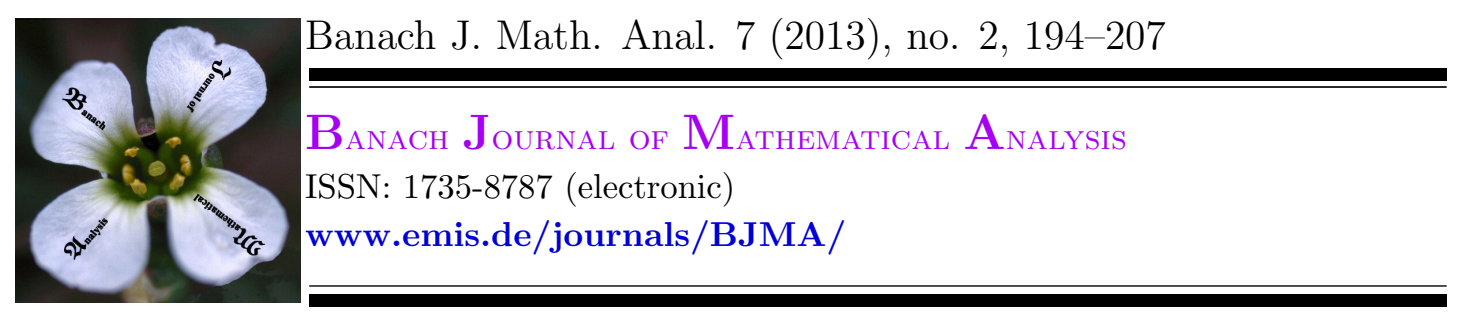

\title{
ON SELFADJOINT DILATION OF THE DISSIPATIVE EXTENSION OF A DIRECT SUM DIFFERENTIAL OPERATOR
}

\author{
EKIN UGURLU ${ }^{1 *}$ AND BILENDER P. ALLAHVERDIEV ${ }^{2}$ \\ Communicated by L. Castro
}

\begin{abstract}
In this paper, we describe all maximal dissipative, maximal accretive and selfadjoint extensions of the minimal symmetric direct sum differential operators. Further using the equivalence of the Lax-Phillips scattering function and the Sz.-Nagy-Foias characteristic function we show that all eigen and associated functions of the maximal dissipative extension of the minimal symmetric direct sum operator are complete in $L_{w}^{2}(\Omega)$, where $\Omega=\Omega_{1} \cup \Omega_{2}, \Omega_{1}=(0, c)$ and $\Omega_{2}=(c, \infty)$.
\end{abstract}

\section{INTRODUCTION}

In the operator theory, one of the main problems is to describe all the selfadjoint extensions of a given minimal symmetric operator. This problem is closely related with finding the dimension of the extended domain. In fact, in 1910 Weyl showed that [19] at least one of the linearly independent solutions of the equation

$$
-y^{\prime \prime}+q(x) y=\lambda y, x \in[0, \infty),
$$

is in squarely integrable space on $[0, \infty)$. This result comes from the convergence of the corresponding nested circles. These circles either converge to a circle or a point. If the primary case occurs, the operator generated by the above differential expression is said to be of limit-circle case, that is, two linearly independent solutions and any combinations of them belong to the squarely integrable space.

Date: Received: 12 July 2012; Revised: 2 January 2013; Accepted: 8 February 2013.

* Corresponding author.

2010 Mathematics Subject Classification. Primary 34B20; Secondary 47E05, 34B24, 34B37.

Key words and phrases. Direct sum differential operators, scattering matrix, characteristic function, spectral analysis. 
Otherwise, the operator is said to be of limit-point case. Of course, a regular differential operator is in limit-circle case. But a singular differential operator may be in limit-point case. These analysis have been used to describe all the selfadjoint extensions of a minimal symmetric differential operators (see [7, 12, 13]). Namely, to describe the selfadjoint extensions of the minimal symmetric differential operators, one can use the defect numbers of the minimal operator $L_{0}$,

$$
m=\operatorname{dim}\left(\left(L_{0}-\bar{\lambda} I\right) D\left(L_{0}\right)\right)^{\perp}, n=\operatorname{dim}\left(\left(L_{0}-\lambda I\right) D\left(L_{0}\right)\right)^{\perp},
$$

where $D\left(L_{0}\right)$ is the domain of $L_{0}$ and $\lambda$ is the complex number. These numbers $(m, n)$ are called the deficiency indices of $L_{0}$. It is well known that $L_{0}$ is selfadjoint if and only if $m=n=0$ and $L_{0}$ has selfadjoint extensions if and only if $m=n$. $(1,1)$ is known as Weyl's limit-point case and $(2,2)$ is known as Weyl's limit-circle case for a second order operator. These are the Weyl's alternatives.

On the other hand in Physics literature such operators having a singularity in the interior of an interval have been investigated [3, 5]. In this case the operators may be handled as direct sum of two operators such that one is defined on one interval and the other is defined on the other interval. Then one may ask that what the deficiency indices of the minimal symmetric direct sum operator are. This question was answered in the paper of Everitt and Zettl [4] (further see [20]). Everitt and Zettl generalized the results known for the deficiency indices theory of the minimal symmetric operators defined on a single interval to two intervals. They characterized all the selfadjoint extensions of a minimal symmetric direct sum operator.

In 1984, Mr. and Mrs. Gorbachuk [8] introduced the method how the nonselfadjoint extensions of the minimal symmetric operator can be given. In fact, let $\Gamma_{1}$ and $\Gamma_{2}$ be the linear mappings of $D\left(A^{*}\right)$ into the Hilbert space $S$ where $A$ is a closed symmetric operator with equal deficiency indices acts in the Hilbert space $S_{1}$. Then $\left(S, \Gamma_{1}, \Gamma_{2}\right)$ is called the space of the boundary values of the operator $A$ if

$i)$ for any $f, g \in D\left(A^{*}\right),\left(A^{*} f, g\right)_{S_{1}}-\left(f, A^{*} g\right)_{S_{1}}=\left(\Gamma_{1} f, \Gamma_{2} g\right)_{S}-\left(\Gamma_{2} f, \Gamma_{1} g\right)_{S}$;

ii) for every $F_{1}, F_{2} \in S$, there exists a vector $f \in D\left(A^{*}\right)$ such that $\Gamma_{1} f=F_{1}$ and $\Gamma_{2} f=F_{2}$.

Then they introduced the following theorem.

Theorem 1.1. [8] For any contraction $K$ in $S$ the restriction of the operator $A^{*}$ to the set of functions $f \in D\left(A^{*}\right)$ satisfying the boundary condition

$$
(K-I) \Gamma_{1} f+i(K+I) \Gamma_{2} f=0,
$$

or

$$
(K-I) \Gamma_{1} f-i(K+I) \Gamma_{2} f=0
$$

is respectively, a maximal dissipative or a maximal accretive extension of the operator $A$, where $A$ is the restriction of the operator $A^{*}$ to the domain $D(A)$. Conversely, every maximal dissipative (maximal accretive) extension of $A$ is the restriction of $A^{*}$ to the set of vectors $f \in D\left(A^{*}\right)$ satisfying (1.1) ((1.2)), and the 
contraction $K$ is uniquely determined by the extension. These conditions give selfadjoint extension if $K$ is unitary. In the latter case (1.1) and (1.2) are equivalent to the condition $(\cos C) \Gamma_{2} f-(\sin C) \Gamma_{1} f=0$, where $C$ is a selfadjoint operator (hermitian matrix) on $S$. The general form of the dissipative and accretive extensions of the operator $A$ is given by the conditions

$$
\begin{aligned}
& K\left(\Gamma_{1} f+i \Gamma_{2} f\right)=\Gamma_{1} f-i \Gamma_{2} f, \Gamma_{1} f+i \Gamma_{2} f \in D(K), \\
& K\left(\Gamma_{1} f-i \Gamma_{2} f\right)=\Gamma_{1} f+i \Gamma_{2} f, \Gamma_{1} f-i \Gamma_{2} f \in D(K),
\end{aligned}
$$

respectively, where $K$ is a linear operator satisfying $\|K f\| \leq\|f\|, f \in D(K)$. The general form of symmetric extensions is given by the formula (1.3) and (1.4), where $K$ is an isometric operator.

It is well known that all eigenvalues of a dissipative operator lie in the closed upper half-plane. But this analysis is so weak. To complete the analysis, there are some methods. One of them is related with the equivalence of the Lax-Phillips scattering function [11] and Sz.-Nagy-Foiaş characteristic function [17]. In the basic of the Lax-Phillips scattering theory there is a decomposition of the Hilbert space $\mathbf{H}$ as $\mathbf{H}=D_{-} \oplus H \oplus D_{+}$in which an unitary group $U_{t}$ has typical properties

$\left.i^{\prime}\right) U_{t} D_{-} \subset D_{-}, t \leq 0 ; U_{t} D_{+} \subset D_{+}, t \geq 0$,

$\left.i i^{\prime}\right) \overline{\bigcup_{t \geq 0} U_{t} D_{-}}=\overline{\bigcup_{t \leq 0} U_{t} D_{+}}=\mathbf{H}$,

iii $\bigcap^{\prime} \bigcap_{t \leq 0} U_{t} D_{-}=\bigcap_{t \geq 0}^{-} U_{t} D_{+}=\{0\}$,

$\left.i v^{\prime}\right) D_{-} \perp D_{+}$,

where the subspaces $D_{-}$and $D_{+}$are called the incoming and outgoing spaces, respectively. Then one can construct the scattering function acting from $D_{+}$to $D_{-}$. On the other hand, Sz.-Nagy and Foiaş defined the characteristic function of a contractive operator $T$ as

$$
\Theta(\mu)=-T+\mu D_{T^{*}}\left(I-\mu T^{*}\right)^{-1} D_{T},
$$

where $\mu$ is some complex number and $D_{T}=\left(I-T^{*} T\right)^{\frac{1}{2}}$ and $D_{T^{*}}=\left(I-T T^{*}\right)^{\frac{1}{2}}$ are the operators defined on some Hilbert space $\widetilde{H}$. There is an equivalence between the Lax-Phillips scattering function and Sz.-Nagy-Foiaş characteristic function (see [17], p. 280). This equivalence has been used in the papers [1] and [2] to analyze the dissipative operators which are the extensions of the minimal symmetric differential operators defined on the single interval.

In this paper we describe all the nonselfadjoint (dissipative, accretive) and selfadjoint extensions of the minimal symmetric direct sum differential operator. In particular, we consider the maximal dissipative extension of the minimal symmetric Bessel-type operator. We construct a selfadjoint dilation of the maximal dissipative extension. To analyze this dissipative operator, we use the functional model theory [17]. After showing that the characteristic function (scattering matrix) given in the model is a Blaschke-Potapov product, we prove that all root (eigen and associated) functions of the dissipative operator which is the extension of the minimal symmetric direct sum operator are complete in the Hilbert space $L_{w}^{2}(\Omega)$, where $\Omega=\Omega_{1} \cup \Omega_{2}, \Omega_{1}=(0, c)$ and $\Omega_{2}=(c, \infty)$. 


\section{MAXimal Dissipative OPERATOR}

In this paper we consider the differential expression

$$
\ell(y):=\frac{1}{w(x)}\left[-y^{\prime \prime}+\frac{\nu^{2}-\frac{1}{4}}{x^{2}} y+q(x) y\right], x \in \Omega:=\Omega_{1} \cup \Omega_{2},
$$

where $\Omega_{1}:=(0, c), \Omega_{2}:=(c, \infty), 0<c<\infty$ and $0 \leq \nu<1$. Throughout the paper it is assumed that the points $0, c$ and $\infty$ are singular for the differential expression $\ell$. Further we assume that $w$ and $q$ are real-valued, Lebesgue measurable functions on $\Omega$, locally integrable functions on $\Omega_{1}$ and $\Omega_{2}$ and $w(x)>0$ for almost all $x \in \Omega$.

Let $H:=H_{1} \oplus H_{2}$, where $H_{1}:=L_{w_{1}}^{2}\left(\Omega_{1}\right), H_{2}:=L_{w_{2}}^{2}\left(\Omega_{2}\right), w(x)=\left\{\begin{array}{l}w_{1}(x), x \in \Omega_{1} \\ w_{2}(x), x \in \Omega_{2}\end{array}\right.$ and $H=L_{w}^{2}(\Omega)$, be the Hilbert space consisting of all complex-valued functions $y$ such that $\int_{\Omega} w(x)|y(x)|^{2} d x<\infty$ with the inner product

$$
(y, \chi)_{H}=(y, \chi)_{H_{1}}+(y, \chi)_{H_{2}}
$$

where

$$
(y, \chi)_{H_{k}}=\int_{\Omega_{k}} w_{k}(x) y_{k}(x) \overline{\chi_{k}(x)} d x
$$

$k=1,2$ and

$$
y(x)=\left\{\begin{array}{l}
y_{1}(x), x \in \Omega_{1} \\
y_{2}(x), x \in \Omega_{2}
\end{array} \in H, \quad \chi(x)=\left\{\begin{array}{l}
\chi_{1}(x), x \in \Omega_{1} \\
\chi_{2}(x), x \in \Omega_{2}
\end{array} \in H .\right.\right.
$$

Let $\Lambda=\Lambda_{1} \oplus \Lambda_{2}$ denote the set of all functions $y \in H$ such that $y^{\prime}$ are locally absolutely continuous functions on $\Omega_{1}$ and $\Omega_{2}$ and $\ell(y) \in H$. The operator $L=L_{1} \oplus L_{2}$ defined on $\Lambda$ by the equality $L y=\ell(y)(x \in \Omega)$ is called the maximal operator [4].

For all $y, \chi \in \Lambda$, Green's formula is obtained as

$$
(\ell(y), \chi)_{H}-(y, \ell(\chi))_{H}=[y, \chi]_{c-}-[y, \chi]_{0}+[y, \chi]_{\infty}-[y, \chi]_{c+},
$$

where $[y, \chi]_{x}:=y(x) \overline{\chi^{\prime}(x)}-y^{\prime}(x) \overline{\chi(x)}(x \in \Omega)$. Green's formula implies that for all functions $y, \chi \in \Lambda$ at singular points $0, c$ and $\infty$, the limits $[y, \chi]_{0}:=$ $\lim _{x \rightarrow 0^{+}}[y, \chi]_{x},[y, \chi]_{c \pm}:=\lim _{x \rightarrow c \pm}[y, \chi]_{x}$ and $[y, \chi]_{\infty}:=\lim _{x \rightarrow+\infty}[y, \chi]_{x}$ exist and are finite.

Let $\Lambda_{0}=\Lambda_{0,1} \oplus \Lambda_{0,2}$ be the set of all functions $y \in \Lambda$ satisfying the conditions

$$
[y, \chi]_{c-}-[y, \chi]_{0}=0,[y, \chi]_{\infty}-[y, \chi]_{c+}=0,
$$

for arbitrary $\chi \in \Lambda$. The operator $L_{0}=L_{0,1} \oplus L_{0,2}$ the restriction of the operator $L=L_{1} \oplus L_{2}$ to the set $\Lambda_{0}$ is called the minimal symmetric operator with the deficiency indices $(r, r)(0 \leq r \leq 4)$ and the equality $L_{0}^{*}=L$ holds [4].

We assume that $w$ and $q$ satisfy the Weyl's limit-circle case conditions at singular points $0, c$ and $\infty$. This means that the deficiency indices of the operator 
$L_{0}$ are $(4,4)$. Weyl's limit point/circle theory is well known and there are several sufficient conditions in which Weyl's limit-circle case holds for a differential expression [9, 12, 18, 20].

Consider the solutions

$$
u(x)=\left\{\begin{array}{l}
u_{1}(x), x \in \Omega_{1} \\
u_{2}(x), x \in \Omega_{2}
\end{array}, v(x)=\left\{\begin{array}{l}
v_{1}(x), x \in \Omega_{1} \\
v_{2}(x), x \in \Omega_{2}
\end{array}\right.\right.
$$

of the equation $\ell(y)=0(x \in \Omega)$ satisfying the initial conditions

$$
\left\{\begin{array} { l l } 
{ u _ { 1 } ( k ) = 1 , } & { u _ { 1 } ^ { \prime } ( k ) = 0 , } \\
{ v _ { 1 } ( k ) = 0 , } & { v _ { 1 } ^ { \prime } ( k ) = 1 , }
\end{array} \quad \left\{\begin{array}{ll}
u_{2}(l)=1, & u_{2}^{\prime}(l)=0, \\
v_{2}(l)=0, & v_{2}^{\prime}(l)=1,
\end{array}\right.\right.
$$

where $k \in \Omega_{1}$ and $l \in \Omega_{2}$. We note that $[u, v]_{x}=1(x \in \Omega)$.

Let $\Gamma_{1}$ and $\Gamma_{2}$ be the linear mappings from $\Lambda$ into $E:=\mathbb{C}^{4}$ as

where

$$
\Gamma_{1} y=\left(\begin{array}{c}
\Gamma_{1}^{-} y \\
\Gamma_{1}^{+} y
\end{array}\right), \quad \Gamma_{2} y=\left(\begin{array}{c}
\Gamma_{2}^{-} y \\
\Gamma_{2}^{+} y
\end{array}\right),
$$

$$
\Gamma_{1}^{-} y:=\left(\begin{array}{l}
{[y, v]_{0}} \\
{[y, u]_{c-}}
\end{array}\right), \quad \Gamma_{1}^{+} y:=\left(\begin{array}{l}
{[y, v]_{c+}} \\
{[y, u]_{\infty}}
\end{array}\right)
$$

and

$$
\Gamma_{2}^{-} y:=\left(\begin{array}{l}
{[y, u]_{0}} \\
{[y, v]_{c-}}
\end{array}\right), \quad \Gamma_{2}^{+} y:=\left(\begin{array}{c}
{[y, u]_{c+}} \\
{[y, v]_{\infty}}
\end{array}\right) .
$$

Lemma 2.1. For any complex numbers $\alpha_{k}, \beta_{k}, \gamma_{k}, \theta_{k}(k=1,2)$ there is a function $y \in \Lambda$ satisfying the conditions

$$
\begin{array}{llll}
{[y, u]_{0}=\alpha_{1},} & {[y, v]_{0}=\alpha_{2},} & {[y, u]_{c-}=\beta_{1},} & {[y, v]_{c-}=\beta_{2},} \\
{[y, u]_{c+}=\gamma_{1},} & {[y, v]_{c+}=\gamma_{2},} & {[y, u]_{\infty}=\theta_{1},} & {[y, v]_{\infty}=\theta_{2} .}
\end{array}
$$

Proof. Let us denote by $L_{0, k}^{-}\left(L_{k}^{-}\right)$and $L_{0, k}^{+}\left(L_{k}^{+}\right)$the minimal (maximal) operators generated by the differential expression $\ell$ on the intervals $I_{k}^{-}$and $I_{k}^{+}$respectively and $\Lambda_{0, k}^{\mp}\left(\Lambda_{k}^{\mp}\right)$ are the domains of $L_{0, k}^{\mp}\left(L_{k}^{\mp}\right)$, where $k=1,2$ and

$$
\begin{aligned}
& I_{1}^{-}=(0, d], I_{1}^{+}=[d, c), 0<d<c, \\
& I_{2}^{-}=(c, e], I_{2}^{+}=[e, \infty), c<e<\infty .
\end{aligned}
$$

We first consider the equation $\ell(y)=\lambda y, x \in(0, c)$. It is known [2] that there is a function $y_{1}^{+} \in \Lambda_{1}^{+}$satisfying the conditions

$$
y_{1}^{+}(d)=\eta_{1}, y_{1}^{+\prime}(d)=\eta_{2},\left[y_{1}^{+}, u\right]_{c-}=\beta_{1},\left[y_{1}^{+}, v\right]_{c-}=\beta_{2},
$$

where $\eta_{1}$ and $\eta_{2}$ are complex numbers. Similarly there is a function $y_{1}^{-} \in \Lambda_{1}^{-}$ satisfying the conditions

$$
y_{1}^{-}(d)=\eta_{1}, y_{1}^{-1}(d)=\eta_{2},\left[y_{1}^{-}, u\right]_{0}=\alpha_{1},\left[y_{1}^{-}, v\right]_{0}=\alpha_{2} .
$$

Now let

$$
y_{1}(x)=\left\{\begin{array}{l}
y_{1}^{-}(x), \quad 0<x \leq d \\
y_{1}^{+}(x), d \leq x<c
\end{array} .\right.
$$


Since the function $y_{1}$ is continuous at the point $x=d$, the function $y_{1} \in \Lambda_{1}$ and the first part of the condition (2.1) is satisfied.

Similarly it can be shown that there is a function $y_{2} \in \Lambda_{2}$ satisfying the conditions

$$
\left[y_{2}, u\right]_{c+}=\gamma_{1},\left[y_{2}, v\right]_{c+}=\gamma_{2},\left[y_{2}, u\right]_{\infty}=\theta_{1},\left[y_{2}, v\right]_{\infty}=\theta_{2} .
$$

Since $y(x)=\left\{\begin{array}{ll}y_{1}(x) & , \quad x \in \Omega_{1} \\ y_{2}(x) & , \quad x \in \Omega_{2}\end{array}\right.$, the proof is completed.

Then we have the following theorem.

Theorem 2.2. The triplet $\left(E, \Gamma_{1}, \Gamma_{2}\right)$ is a space of boundary values of the operator $L_{0}$.

Proof. For $y, \chi \in \Lambda$, we have

$$
\left(L_{0}^{*} y, \chi\right)_{H}-\left(y, L_{0}^{*} \chi\right)_{H}=[y, \chi]_{c-}-[y, \chi]_{0}+[y, \chi]_{\infty}-[y, \chi]_{c+} .
$$

On the other hand with a direct calculation we get that

$$
\left(\Gamma_{1} y, \Gamma_{2} \chi\right)_{E}-\left(\Gamma_{2} y, \Gamma_{1} \chi\right)_{E}=[y, \chi]_{c-}-[y, \chi]_{0}+[y, \chi]_{\infty}-[y, \chi]_{c+} .
$$

(2.2) and (2.3) complete the first part $i$ ) given in the introduction of the proof. Lemma 2.1 completes the second part $i i$ ) given in the introduction. Hence the proof is completed.

Then from Theorem 1.1, we can introduce the following theorem.

Theorem 2.3. For $y \in D(L)$, the boundary conditions

$$
\begin{gathered}
{[y, u]_{0}+h_{1}[y, v]_{0}=0} \\
{[y, v]_{c-}+h_{2}[y, u]_{c-}=0,} \\
{[y, u]_{c+}+h_{3}[y, v]_{c+}=0} \\
{[y, v]_{\infty}+h_{4}[y, u]_{\infty}=0,}
\end{gathered}
$$

with $\Im h_{i} \geq 0$ or $h_{i}=\infty(i=1,2,3,4)$ describe all the maximal dissipative extensions of $L_{0}$ with separated boundary conditions.

In this paper we assume that $K$ is a strict contraction in $E$, i.e., $\|K\|_{E}<1$.

Let $\mathcal{L}$ be the operator generated by $\ell$ and the condition

$$
\Gamma_{2} y+R \Gamma_{1} y=0,
$$

where $R=-i(K+I)^{-1}(K-I), \Im R>0$, and $-K$ is the Cayley transform of the dissipative operator $R$.

If $R$ is chosen as

$$
R=\left(\begin{array}{cccc}
h_{1} & & & 0 \\
& h_{2} & & \\
& & h_{3} & \\
0 & & & h_{4}
\end{array}\right)
$$


where $\Im h_{i}>0(i=1,2,3,4)$, then the boundary condition (2.8) coincides with the separated boundary conditions (2.4)-(2.7).

We note that $\mathcal{L}$ is the maximal dissipative operator in $H$.

\section{Characteristic function of $\mathcal{L}$}

Let us consider the Hilbert space $\mathbf{H}=L^{2}\left(\mathbb{R}_{-} ; E\right) \oplus H \oplus L^{2}\left(\mathbb{R}_{+} ; E\right)$, where $\mathbb{R}_{-}:=(-\infty, 0]$ and $\mathbb{R}_{+}:=[0, \infty)$. The spaces $L^{2}\left(\mathbb{R}_{-} ; E\right)$ and $L^{2}\left(\mathbb{R}_{+} ; E\right)$ are called the incoming and outgoing channels, respectively.

We denote by $\boldsymbol{\Lambda}$ the set consisting of all functions $f=\left\langle\varphi_{-}, y, \varphi_{+}\right\rangle \in \mathbf{H}$, where $\varphi_{-} \in W_{2}^{1}\left(\mathbb{R}_{-} ; E\right), y \in \Lambda$ and $\varphi_{+} \in W_{2}^{1}\left(\mathbb{R}_{+} ; E\right)$ ( $W_{2}^{1}$ is the Sobolev space) satisfying the conditions

$$
\Gamma_{2} y+R \Gamma_{1} y=C \varphi_{-}(0), \quad \Gamma_{2} y+R^{*} \Gamma_{1} y=C \varphi_{+}(0),
$$

where $C^{2}:=2 \Im R, C>0$.

We consider the operator $\aleph$ on the set $\boldsymbol{\Lambda}$ generated by the differential expression

$$
\widetilde{\aleph}\left\langle\varphi_{-}, y, \varphi_{+}\right\rangle=\left\langle i \frac{d \varphi_{-}}{d \xi}, \ell(y), i \frac{d \varphi_{+}}{d \zeta}\right\rangle
$$

as $\aleph f=\widetilde{\aleph} f$. Then we have the following theorem.

Theorem 3.1. The operator $\aleph$ is selfadjoint in $\mathbf{H}$.

Proof. For $f=\left\langle\varphi_{-}, y, \varphi_{+}\right\rangle, g=\left\langle\psi_{-}, \chi, \psi_{+}\right\rangle \in \boldsymbol{\Lambda}$, we have

$$
\begin{aligned}
(\aleph f, g)_{\mathbf{H}}-(f, \aleph g)_{\mathbf{H}}= & {[y, \chi]_{c-}-[y, \chi]_{0}+[y, \chi]_{\infty}-[y, \chi]_{c+} } \\
& +i\left(\varphi_{-}(0), \psi_{-}(0)\right)_{E}-i\left(\varphi_{+}(0), \psi_{+}(0)\right)_{E} .
\end{aligned}
$$

Using the condition (3.1) one gets

$$
i\left(\varphi_{-}(0), \psi_{-}(0)\right)_{E}-i\left(\varphi_{+}(0), \psi_{+}(0)\right)_{E}=\left(\Gamma_{2} y, \Gamma_{1} \chi\right)_{E}-\left(\Gamma_{1} y, \Gamma_{2} \chi\right)_{E} .
$$

Substituting (3.4) in (3.3) we obtain that $\aleph$ is symmetric in $\mathbf{H}$.

Now let $f=\left\langle\varphi_{-}, 0, \varphi_{+}\right\rangle \in \boldsymbol{\Lambda}, \varphi_{\mp} \in W_{2}^{1}\left(\mathbb{R}_{\mp} ; E\right), \varphi_{\mp}(0)=0$. Then for $g=$ $\left\langle\psi_{-}, \chi, \psi_{+}\right\rangle \in \boldsymbol{\Lambda}$ we have

$$
\begin{aligned}
(\aleph f, g)_{\mathbf{H}} & =\left(\left\langle i \frac{d \varphi_{-}}{d \xi}, 0, i \frac{d \varphi_{+}}{d \zeta}\right\rangle,\left\langle\psi_{-}, \chi, \psi_{+}\right\rangle\right)_{\mathbf{H}} \\
& =\left(\left\langle\varphi_{-}, 0, \varphi_{+}\right\rangle,\left\langle i \frac{d \psi_{-}}{d \xi}, \chi^{*}, i \frac{d \psi_{+}}{d \zeta}\right\rangle\right)_{\mathbf{H}} .
\end{aligned}
$$

This implies that $\aleph^{*} g=\left\langle i \frac{d \psi_{-}}{d \xi}, \chi^{*}, i \frac{d \psi_{+}}{d \zeta}\right\rangle$, where $\psi_{\mp} \in W_{2}^{1}\left(\mathbb{R}_{\mp}\right), \chi^{*} \in H$.

Again let $f=\langle 0, y, 0\rangle \in \boldsymbol{\Lambda}$ and put it in (3.5). Then we arrive at

$$
\aleph^{*} g=\left\langle i \frac{d \psi_{-}}{d \xi}, \ell(\chi), i \frac{d \psi_{+}}{d \zeta}\right\rangle, \chi \in \Lambda .
$$

Consequently we obtain

$$
[y, \chi]_{c-}-[y, \chi]_{0}+[y, \chi]_{\infty}-[y, \chi]_{c+}+i\left(\varphi_{-}(0), \psi_{-}(0)\right)_{E}-i\left(\varphi_{+}(0), \psi_{+}(0)\right)_{E}=0 \text {. }
$$


Further solving the boundary conditions (3.1) we find that

$$
\begin{aligned}
& \Gamma_{1} y=-i C^{-1}\left[\varphi_{-}(0)-\varphi_{+}(0)\right], \\
& \Gamma_{2} y=C \varphi_{-}(0)+i R C^{-1}\left[\varphi_{-}(0)-\varphi_{+}(0)\right] .
\end{aligned}
$$

Using (3.6) and (3.7) one gets that

$$
\begin{aligned}
& i\left(\varphi_{-}(0), \psi_{-}(0)\right)_{E}-i\left(\varphi_{+}(0), \psi_{+}(0)\right)_{E}=\left(\Gamma_{2} y, \Gamma_{1} \chi\right)_{E}-\left(\Gamma_{1} y, \Gamma_{2} \chi\right)_{E} \\
& =i\left(\varphi_{-}(0), i C \Gamma_{1} \chi+C^{-1} R^{*} \Gamma_{1} \chi+C^{-1} \Gamma_{2} \chi\right)_{E}-i\left(\varphi_{+}(0), C^{-1} R^{*} \Gamma_{1} \chi+C^{-1} \Gamma_{2} \chi\right)_{E} .
\end{aligned}
$$

Since the values $\varphi_{\mp}(0)$ can be arbitrary vectors, comparing the coefficients of $\varphi_{\mp}(0)$, on the left and right of the equality (3.8) it is obtained that

$$
\Gamma_{2} \chi+R \Gamma_{1} \chi=C \psi_{-}(0), \quad \Gamma_{2} \chi+R^{*} \Gamma_{1} \chi=C \psi_{+}(0) .
$$

This implies that $D\left(\aleph^{*}\right) \subseteq D(\aleph)$, and hence $\aleph=\aleph^{*}$.

Let us consider the operator family $Z_{t}:=P U_{t} P_{1}, t \geq 0$, where $U_{p}:=\exp (i \aleph p)$ $(p \in \mathbb{R})$ is a unitary group and $P$ and $P_{1}$ are the linear mappings as

$$
\begin{aligned}
& P: \mathbf{H} \quad \rightarrow H \quad P_{1}: H \rightarrow \mathbf{H} \\
& \left\langle\varphi_{-}, y, \varphi_{+}\right\rangle \rightarrow y, \quad y \rightarrow\langle 0, y, 0\rangle \text {. }
\end{aligned}
$$

It is known that the operator family $\left\{Z_{t}\right\}(t \geq 0)$ is the strongly continuous semigroup of completely nonunitary contractions on $\mathbf{H}[1,2,14,15]$.

Let $G$ denote the generator of the semigroup $Z_{t}$

$$
G y=\lim _{t \rightarrow+0} \frac{1}{i t}\left(Z_{t} y-y\right) \text {. }
$$

The domain of $G$ consists of all the vectors for which the limit exists. The operator $G$ is a maximal dissipative operator $[1,2,14,15]$ and $\aleph$ is called the selfadjoint dilation of $G[10,17]$.

Theorem 3.2. The operator $\aleph$ is a selfadjoint dilation of the operator $\mathcal{L}$.

Proof. For $y \in H, g \in \boldsymbol{\Lambda}$ and $\Im \lambda<0$, let us consider the equality

$$
(\aleph-\lambda I)^{-1} P_{1} y=g=\left\langle\psi_{-}, \chi, \psi_{+}\right\rangle .
$$

(3.9) is also equivalent to the equality $(\aleph-\lambda I) g=P_{1} y$. Therefore we have $\ell(\chi)-$ $\lambda \chi=y, \psi_{-}(\xi)=\psi_{-}(0) e^{-i \lambda \xi}$ and $\psi_{+}(\zeta)=\psi_{+}(0) e^{-i \lambda \zeta}$. Since $\psi_{-} \in L^{2}\left(\mathbb{R}_{-} ; E\right)$ we get that $\psi_{-}(0)=0$. Hence $\chi$ satisfies the condition $\Gamma_{2} \chi+R \Gamma_{1} \chi=0$. Further since a value $\lambda$ with $\Im \lambda<0$ can not be an eigenvalue of a dissipative operator, one obtains $\chi=(\mathcal{L}-\lambda I)^{-1} y$. So we arrive at

$$
(\aleph-\lambda I)^{-1} P_{1} y=\left\langle 0,(\mathcal{L}-\lambda I)^{-1} y, C^{-1}\left(\Gamma_{2} \chi+R^{*} \Gamma_{1} \chi\right) e^{-i \lambda \zeta}\right\rangle .
$$

Applying the mapping $P$ to (3.10), we get that

$$
P(\aleph-\lambda I)^{-1} P_{1} y=(\mathcal{L}-\lambda I)^{-1} y .
$$

On the other hand the equalities

$$
P(\aleph-\lambda I)^{-1} P_{1}=-i P \int_{0}^{\infty} U_{t} e^{-i \lambda t} d t P_{1}=-i \int_{0}^{\infty} Z_{t} e^{-i \lambda t} d t=(G-\lambda I)^{-1}
$$


hold. (3.11) and (3.12) complete the proof.

We shall remind that the linear operator $B$ (with domain $D(B)$ ) acting in the Hilbert space $S$ is called completely nonselfadjoint (or simple) if there is no invariant subspace $M \subseteq D(B)(M \neq\{0\})$ of the operator $B$ on which the restriction $B$ on $M$ is selfadjoint.

Lemma 3.3. The operator $\mathcal{L}$ is completely nonselfadjoint (simple) in the Hilbert space $H$.

Proof. Let $\widetilde{\mathcal{L}}$ be the selfadjoint part of $\mathcal{L}$ in the subspace $\widetilde{H} \subset H$ with domain $D(\widetilde{\mathcal{L}})=\widetilde{H} \cap D(\mathcal{L})$. For $y \in D(\widetilde{\mathcal{L}})$ (also for $y \in D\left(\widetilde{\mathcal{L}}^{*}\right)$ )

$$
\begin{aligned}
0 & =(\widetilde{\mathcal{L}} y, y)_{H}-(y, \widetilde{\mathcal{L}} y)_{H}=\left(\Gamma_{1} y, \Gamma_{2} y\right)_{E}-\left(\Gamma_{2} y, \Gamma_{1} y\right)_{E} \\
& =\left(\Gamma_{1} y,-R \Gamma_{1} y\right)_{E}-\left(-R \Gamma_{1} y, \Gamma_{1} y\right)_{E}=\left(\left(R-R^{*}\right) \Gamma_{1} y, \Gamma_{1} y\right)_{E} \\
& =2 i\left(\Im R \Gamma_{1} y, \Gamma_{1} y\right)_{E} .
\end{aligned}
$$

This implies that $\Gamma_{1} y=0$. From (2.8) we get that $\Gamma_{2} y=0$. Hence $y \equiv 0$. By the theorem on expansion in eigenfunctions of the selfadjoint operator $\widetilde{\mathcal{L}}$ we have $\widetilde{H}=\{0\}$. This completes the proof.

Let $D_{-}=\left\langle L^{2}\left(\mathbb{R}_{-} ; E\right), 0,0\right\rangle$ and $D_{+}=\left\langle 0,0, L^{2}\left(\mathbb{R}_{+} ; E\right)\right\rangle$. According to the Lax-Phillips scattering theory [11], the unitary group $U_{t}$ and the spaces $D_{-}$and $D_{+}$satisfy the properties $\left.i^{\prime}\right)-i v^{\prime}$ ) given in the introduction. To see that these properties are satisfied, see the detailed proofs in $[1,2,14,15]$.

Consider the solutions

$$
\varphi(x, \lambda)=\left\{\begin{array}{l}
\varphi_{1}(x, \lambda), x \in \Omega_{1} \\
\varphi_{2}(x, \lambda), x \in \Omega_{2}
\end{array}, \quad \psi(x, \lambda)=\left\{\begin{array}{l}
\psi_{1}(x, \lambda), x \in \Omega_{1} \\
\psi_{2}(x, \lambda), x \in \Omega_{2}
\end{array},\right.\right.
$$

of $\ell(y)=\lambda y(x \in \Omega)$ satisfying the initial conditions

$$
\left\{\begin{array} { l l } 
{ [ \varphi _ { 1 } , v _ { 1 } ] _ { 0 } = 0 , } & { [ \varphi _ { 1 } , u _ { 1 } ] _ { 0 } = - 1 , } \\
{ [ \psi _ { 1 } , v _ { 1 } ] _ { 0 } = 1 , } & { [ \psi _ { 1 } , u _ { 1 } ] _ { 0 } = 0 , }
\end{array} \quad \left\{\begin{array}{ll}
{\left[\varphi_{2}, v_{2}\right]_{c+}=0,} & {\left[\varphi_{2}, u_{2}\right]_{c+}=-1,} \\
{\left[\psi_{2}, v_{2}\right]_{c+}=1,} & {\left[\psi_{2}, u_{2}\right]_{c+}=0 .}
\end{array}\right.\right.
$$

Let $M_{1}(\lambda)$ and $M_{2}(\lambda)$ be the matrix-valued functions satisfying the conditions

$$
\left\{\begin{array}{l}
M_{1}(\lambda) \Gamma_{1}^{-} \varphi_{1}=\Gamma_{2}^{-} \varphi_{1} \\
M_{1}(\lambda) \Gamma_{1}^{-} \psi_{1}=\Gamma_{2}^{-} \psi_{1}
\end{array}, \quad\left\{\begin{array}{l}
M_{2}(\lambda) \Gamma_{1}^{+} \varphi_{2}=\Gamma_{2}^{+} \varphi_{2} \\
M_{2}(\lambda) \Gamma_{1}^{+} \psi_{2}=\Gamma_{2}^{+} \psi_{2}
\end{array} .\right.\right.
$$

It is possible to find the entries of $M_{1}(\lambda)$ and $M_{2}(\lambda)$. Using these matrix-valued functions we construct the matrix-valued function $M(\lambda)$ as

Then we have

$$
M(\lambda)=\left(\begin{array}{cc}
M_{1}(\lambda) & 0 \\
0 & M_{2}(\lambda)
\end{array}\right) .
$$

$$
M(\lambda) \Gamma_{1} \varphi=\Gamma_{2} \varphi, \quad M(\lambda) \Gamma_{1} \psi=\Gamma_{2} \psi .
$$

$M(\lambda)$ has the following properties

$\left.i^{\prime \prime}\right) M(\lambda)$ is meromorphic in $\mathbb{C}$ with all its poles on real axis $\mathbb{R}$, 
$\left.i i^{\prime \prime}\right) \Im M(\lambda) \leq 0$ for $\Im \lambda>0$,

$\left.i i i^{\prime \prime}\right) \Im M(\lambda) \geq 0$ for $\Im \lambda<0$,

$\left.i v^{\prime \prime}\right) M^{*}(\lambda)=M(\lambda)$ for all $\lambda \in \mathbb{R}$, except for the poles of $M(\lambda)$.

Let

$$
\eta_{j}:=\eta_{j}(x, \lambda)=\left\{\begin{array}{l}
\eta_{j, 1}(x, \lambda), x \in \Omega_{1} \\
\eta_{j, 2}(x, \lambda), x \in \Omega_{2}
\end{array}, \theta_{j}:=\theta_{j}(x, \lambda)=\left\{\begin{array}{c}
\theta_{j, 1}(x, \lambda), x \in \Omega_{1} \\
\theta_{j, 2}(x, \lambda), x \in \Omega_{2}
\end{array}\right.\right.
$$

be the solutions of the equation $\ell(y)=\lambda y(x \in \Omega)$ satisfying the conditions

$$
\begin{aligned}
& \Gamma_{1} \eta_{j}=(M(\lambda)+R)^{-1} C e_{j}, \\
& \Gamma_{1} \theta_{j}=\left(M(\lambda)+R^{*}\right)^{-1} C e_{j},
\end{aligned}
$$

where $j=1,2,3,4$, and $e_{j}$ are the orthonormal basis for $E$.

Now consider the functions $\pi_{\lambda, j}^{-}:=\pi_{\lambda, j}^{-}(x, \xi, \zeta)$ and $\pi_{\lambda, j}^{+}:=\pi_{\lambda, j}^{+}(x, \xi, \zeta)$ as

$$
\begin{gathered}
\pi_{\lambda, j}^{-}=\left\langle e^{-i \lambda \xi} e_{j}, \eta_{j}, C^{-1}\left(M(\lambda)+R^{*}\right)(M(\lambda)+R)^{-1} C e^{-i \lambda \zeta} e_{j}\right\rangle, \\
\pi_{\lambda, j}^{+}=\left\langle S(\lambda) e^{-i \lambda \xi} e_{j}, \theta_{j}, e^{-i \lambda \zeta} e_{j}\right\rangle,
\end{gathered}
$$

where $j=1,2,3,4$ and

$$
S(\lambda)=C^{-1}(M(\lambda)+R)\left(M(\lambda)+R^{*}\right)^{-1} C .
$$

For all $\lambda \in \mathbb{R}$, the functions $\pi_{\lambda, j}^{\mp}$ do not belong to the space $\mathbf{H}$ but they satisfy the equation $\aleph \pi_{\lambda, j}^{\mp}=\lambda \pi_{\lambda, j}^{\mp}$. Using these functions we define the transformations $\mathcal{F}_{-}: f \rightarrow \tilde{f}_{-}(\lambda)$ and $\mathcal{F}_{+}: f \rightarrow \tilde{f}_{+}(\lambda)$ as

$$
\left(\mathcal{F}_{\mp} f\right)(\lambda):=\widetilde{f}_{\mp}(\lambda):=\sum_{j=1}^{4} f_{j}^{\mp}(\lambda) e_{j},
$$

where

$$
f_{j}^{\mp}(\lambda)=\frac{1}{\sqrt{2 \pi}}\left(f, \pi_{\lambda, j}^{\mp}\right)_{\mathbf{H}}
$$

and $f=\left\langle\varphi_{-}, y, \varphi_{+}\right\rangle$in which $\varphi_{-}, \varphi_{+}$and $y$ are smooth, compactly supported functions.

Let

$$
H_{-}=\overline{\bigcup_{t \geq 0} U_{t} D_{-}}, H_{+}=\overline{\bigcup_{t \leq 0} U_{t} D_{+}} .
$$

The transformation $\mathcal{F}_{-}$isometrically maps $H_{-}$onto $L^{2}(\mathbb{R} ; E)$ and the transformation $\mathcal{F}_{+}$isometrically maps $H_{+}$onto $L^{2}(\mathbb{R} ; E)$. The detailed proofs were given in $[1,2,14,15]$. Further for all vectors $f, g \in H_{\mp}$, the Parseval equality and the inverse formula hold [1, 2, 14, 15]:

$$
(f, g)_{\mathbf{H}}=\left(\widetilde{f}_{\mp}, \widetilde{g}_{\mp}\right)_{L^{2}}=\int_{-\infty}^{\infty} \sum_{j=1}^{4} f_{j}^{\mp}(\lambda) \overline{g_{j}^{\mp}(\lambda)} d \lambda, \quad f=\frac{1}{\sqrt{2 \pi}} \int_{-\infty}^{\infty} \sum_{j=1}^{4} \pi_{\lambda, j}^{\mp} f_{j}^{\mp}(\lambda) d \lambda .
$$


It is clear that the matrix-valued function $S(\lambda)$ given in (3.13) is meromorphic in $\mathbb{C}$ and all poles are in the lower half-plane. Further it is possible to get that $\|S(\lambda)\| \leq 1$ for $\Im \lambda>0$ and $S(\lambda)$ is the unitary matrix for all $\lambda \in \mathbb{R}$. Then for $\lambda \in \mathbb{R}$ we have

$$
\pi_{\lambda, j}^{+}=\sum_{k=1}^{4} S_{j k}(\lambda) \pi_{\lambda, j}^{-},
$$

where $S_{j k}(\lambda),(j=1,2,3,4)$ are the entries of the matrix $S(\lambda)$.

According to the Lax-Phillips scattering theory [11], $\mathcal{F}_{-}$is the incoming spectral representation for $U_{t}$ and $\mathcal{F}_{+}$is the outgoing spectral representation for $U_{t}$. From (3.14) we get that

$$
\tilde{f}_{-}=S(\lambda) \tilde{f}_{+}
$$

Since the scattering matrix of the group $U_{t}$ with respect to the subspaces $D_{-}$and $D_{+}$is the coefficient by which the $\mathcal{F}_{-}$-representation of a vector $f \in \mathbf{H}$ must be multiplied in order to get the corresponding $\mathcal{F}_{+}$-representation: $\tilde{f}_{+}(\lambda)=$ $S^{-1}(\lambda) \widetilde{f}_{-}(\lambda)$, we have the following.

Theorem 3.4. The matrix $S^{-1}(\lambda)$ is the scattering matrix of the group $U_{t}$.

From Lemma 3.3 one gets that $[1,2,14,15]$

$$
H_{-}+H_{+}=\mathbf{H}
$$

Since the spaces $H_{-}$and $H_{+}$are isometrically identical with $L^{2}(\mathbb{R} ; E)$ we arrive at

$$
H_{-}=H_{+}=\mathbf{H} \text {. }
$$

Hence under the transformation $\mathcal{F}_{-}$we have the following mappings $[1,2,14,15]$

1) $\mathbf{H} \rightarrow L^{2}(\mathbb{R} ; E)$,

2) $f \rightarrow \widetilde{f}_{-}(\lambda)$,

3) $D_{-} \rightarrow H_{-}^{2}$

4) $D_{+} \rightarrow S(\lambda) H_{+}^{2}$

5) $\langle 0, H, 0\rangle \rightarrow H_{+}^{2} \ominus S(\lambda) H_{+}^{2}$,

6) $U_{t} f \rightarrow\left(\mathcal{F}_{-} U_{t} \mathcal{F}_{-}^{-1} \widetilde{f}_{-}\right)(\lambda)=e^{i \lambda t} \widetilde{f}_{-}(\lambda)$,

where $H_{+}^{2}$ is the Hardy class in $L^{2}(\mathbb{R} ; E)$ consisting of the vector valued functions analytically extendible to the upper half-plane. These transformations 1) - 6) show that the maximal dissipative operator $\mathcal{L}$ is unitary equivalent to the model dissipative operator with characteristic function $S(\lambda)[10,15,17]$. Since the characteristic functions of unitary equivalent dissipative operators coincide with each other [17], we can introduce the following theorem.

Theorem 3.5. The characteristic function of the maximal dissipative operator $\mathcal{L}$ coincides with the matrix-valued function $S(\lambda)$. 


\section{The SPECTRAL analysis of the maXimal Dissipative operator $\mathcal{L}$}

Characteristic function can answer the question of whether all eigenfunctions and associated functions of a maximal dissipative operator span the whole space or not. This analysis can be done with ensuring that the singular factor $s(\lambda)$ in the factorization $\operatorname{det} S(\lambda)=s(\lambda) \mathcal{B}(\lambda)(\mathcal{B}(\lambda)$ is the Blaschke product) is absent (see $[10,17])$.

But, before the calculations we need to define a suitable form for the $\Gamma$-capacity $[6,16]$.

Let $\tilde{E}$ be an $m$-dimensional $(m<\infty)$ Euclidean space. In $\tilde{E}$, we fix an orthonormal basis $e_{1}, e_{2}, \ldots, e_{m}$ and denote by $E_{k}(k=1,2, \ldots, m)$ the linear span of vectors $e_{1}, e_{2}, \ldots, e_{k}$. If $M \subset E_{k}$, then the set of $x \in E_{k-1}$ with the property $\operatorname{Cap}\left\{\lambda: \lambda \in \mathbf{C},\left(x+\lambda e_{k}\right) \in M\right\}>0$ will be denoted by $\Gamma_{k-1} M$. (Cap $G$ is the inner logarithmic capacity of the set $G \subset \mathbf{C}$ ). The $\Gamma$-capacity of the set $M \subset \tilde{E}$ is a number $\Gamma-C a p M:=\sup C a p\left\{\lambda: \lambda \in \mathbf{C}, \lambda e_{1} \subset \Gamma_{1} \Gamma_{2} \ldots \Gamma_{m-1} M\right\}$, where the sup is taken with respect to all orthonormal basis in $\tilde{E}$ (see $[6,16])$. It is known that $[6,16]$ every set $M \subset \tilde{E}$ of zero $\Gamma$-capacity has zero $2 m$-dimensional Lebesgue measure (in the decomplexified space $\tilde{E}$ ), however, the converse is false.

Denote by $[E]$ the set of all linear operators in $E\left(=\mathbf{C}^{4}\right)$. To convert $[E]$ into the 16-dimensional Euclidean space, we introduce the inner product $\langle T, S\rangle=\operatorname{tr} S^{*} T$ for $T, S \in[E]\left(\operatorname{tr} S^{*} T\right.$ is the trace of the operator $\left.S^{*} T\right)$. Hence, we may introduce the $\Gamma$-capacity of a set of $[E]$.

To reach our main aim we will use the following lemma (see [2]).

Lemma 4.1. The characteristic function $S(\lambda)$ of the operator $\mathcal{L}$ has the form

$$
S(\lambda)=X_{1}\left(I-K_{1} K_{1}^{*}\right)^{-\frac{1}{2}}\left(\theta(\xi)-K_{1}\right)\left(I-K_{1}^{*} \theta(\xi)\right)^{-1}\left(I-K_{1}^{*} K_{1}\right)^{\frac{1}{2}} X_{2},
$$

where $K_{1}=-K$ is the Cayley transformation of the dissipative operator $R$, and $\theta(\xi)$ is the Cayley transformation of the matrix-valued function $M(\lambda), \xi=$ $(\lambda-i)(\lambda+i)^{-1}$, and

$$
\begin{gathered}
X_{1}:=(\Im R)^{-\frac{1}{2}}\left(I-K_{1}\right)^{-1}\left(I-K_{1} K_{1}^{*}\right)^{\frac{1}{2}} \\
X_{2}:=\left(I-K_{1}^{*} K_{1}\right)^{-\frac{1}{2}}\left(I-K_{1}^{*}\right)(\Im R)^{\frac{1}{2}},\left|\operatorname{det} X_{1}\right|=\left|\operatorname{det} X_{2}\right|=1 .
\end{gathered}
$$

It is known $[6,10]$ that the inner matrix-valued function $S(\lambda)$ is a BlaschkePotapov product if and only if $\operatorname{det} S(\lambda)$ is a Blaschke product. So from Lemma 4.1 we can infer that the characteristic function $S(\lambda)$ is a Blaschke-Potapov product if and only if the matrix-valued function

$$
X_{K}(\xi)=\left(I-K_{1} K_{1}^{*}\right)^{-\frac{1}{2}}\left(\theta(\xi)-K_{1}\right)\left(I-K_{1}^{*} \theta(\xi)\right)^{-1}\left(I-K_{1}^{*} K_{1}\right)^{\frac{1}{2}}
$$

is a Blaschke-Potapov product in a unit disk.

We will utilize the following important result of the paper [6].

Lemma 4.2. Let $X(\xi)(|\xi|<1)$ be a holomorphic function with the values to be contractive operators in $[E]$ (i.e., $\left.\|X(\xi)\|_{E} \leq 1\right)$. Then for $\Gamma$-quasi-every strictly contractive operators $K$ in $[E]$ (i.e., for all strictly contractive $K \in[E]$ with the 
possible exception of a set of $\Gamma$-capacity zero) the inner part of the contractive function

$$
X_{K}(\xi):=\left(I-K K^{*}\right)^{-\frac{1}{2}}(X(\xi)-K)\left(I-K^{*} X(\xi)\right)^{-1}\left(I-K^{*} K\right)^{\frac{1}{2}}
$$

is a Blaschke-Potapov product.

Showing the absence of the singular factor $s(\lambda)$ in the factorization $\operatorname{det} S(\lambda)=$ $s(\lambda) \mathcal{B}(\lambda)(\mathcal{B}(\lambda)$ is the Blaschke-Potapov product) ensures the completeness of the eigenfunctions and associated functions of the operator $\mathcal{L}$ in the space $L_{w}^{2}(\Omega)$ (see $[6,10,15,17])$.

Finally summarizing all the obtained results for the maximal dissipative operators $\mathcal{L}$, we can introduce the following theorem.

Theorem 4.3. For $\Gamma$-quasi-every strictly contractive $K \in[E]$ (i.e., for all strictly contractive $K \in[E]$ with the possible exception of a set of $\Gamma$-capacity zero), the characteristic function $S(\lambda)$ of the maximal dissipative operator $\mathcal{L}$ is a BlaschkePotapov product, and the spectrum of $\mathcal{L}$ is purely discrete and belongs to the open upper half-plane. For $\Gamma$-quasi-every strictly contractive $K \in[E]$, the operator $\mathcal{L}$ has a countable number of isolated eigenvalues with finite multiplicity and limit point at infinity, and the system of eigenfunctions and associated functions of this operator is complete in the space $L_{w}^{2}(\Omega)$.

\section{REFERENCES}

1. B.P. Allahverdiev and A. Canoğlu, Spectral analysis of dissipative Schödinger operators, Proc. Roy. Soc. Edinburgh Sect. A 127 (1997), no. 6, 1113-1121.

2. B.P. Allahverdiev, Dissipative Sturm-Liouville operators with nonseparated boundary conditions, Monatsh. Math. 140 (2003), 1, 1-17.

3. J.P. Boyd, Sturm-Liouville eigenvalue problems with an interior pole, J. Math. Phys. 22 (1981), 8, 1575-1590.

4. W.N. Everitt and A. Zettl, Sturm-Liouville differential operators in direct sum spaces, Rocky Mountain J. Math. 16 (1986), 3, 497-516.

5. F. Gesztesy and W. Kirsch, One-dimensional Schrödinger operators with interactions singular on a discrete set, J. Reine Angew. Math. 362 (1985), 28-50.

6. Yu.P. Ginzburg and N.A. Talyush, Exceptional sets of analytic matrix-functions, contracting and dissipative operators, Izv. Vyssh. Uchebn. Zaved. Mat. 267 (1984), 9-14; English transl. Soviet Math. (Izv. VUZ) 28 (1984), 10-16.

7. I.M. Glazman, Direct Methods of Qualitative Spectral Analysis of Singular Differential Operators, Israeli Program for Scientific Translations, 1965.

8. V.I. Gorbachuk and M.L. Gorbachuk, Boundary Value Problems for Operator Differential Equations, Naukova Dumka, Kiev, 1984; English transl. Kluwer, Dordrecht, 1991.

9. B.J. Harris, Limit-circle criteria for second order differential expression, Quart. J. Math. Oxford 2. Ser. 35 (1984), 415-427.

10. A. Kuzhel, Characteristic Functions and Models of Nonself-adjoint Operators, Kluwer, Dordrecht, 1996.

11. P.D. Lax and R.S. Phillips, Scattering Theory, Academic Press, New York, 1967.

12. M.A. Naimark, Linear Differential Operators, 2nd edn, Nauka, Moscow, 1969; English transl. of 1st edn, Parts 1, 2, Frederick Ungar Publishing Co., New York, 1967, 1968.

13. J. von Neumann, Allgemeine eigenwertheorie Hermitischer functional operatoren, Math. Ann. 102 (1929), 49-131.

14. M.Y. Ongun, Spectral analysis of nonselfadjoint Schörinder problem with eigenparameter in the boundary condition, Sci. China Ser. A 50 (2007), 2, 217-230. 
15. B.S. Pavlov, Spectral analysis of a dissipative singular Schrödinger operator in terms of a functional model, Itogi Nauki Tekh. Ser. Sovrem. Probl. Math. Fundam Napravleniya, 65 (1991), 95-163, English transl. in partial differential equations, 8 Encyc. Math. Sci. 65 (1996), 87-163.

16. L.I. Ronkin, Introduction to the Theory of Entire Functions of Several Variables, Nauka, Moscow, 1971; English transl. Amer. Math. Soc., Providence, RI, 1974.

17. B. Sz.-Nagy and C. Foiaş, Analyse Harmonique des Operateurs de L'espace de Hilbert, Masson, Paris and Akad Kiadó, Budapest, 1967; English transl. North-Holland, Amsterdam and Akad. Kiadó, Budapest, 1970.

18. E.C. Titchmarsh, Eigenfunction Expansions Associated with Second Order Differential Equations, Part 1, Oxford University Press, 2nd edn, 1962.

19. H. Weyl, Über gewöhnliche differentialgleichungen mit singularitäten und die zugehörigen entwicklungen wilkürlichen funktionen, Math. Ann. 68 (1910), 220-269.

20. A. Zettl, Sturm-Liouville Theory, Mathematical Surveys and Monographs, 121, American Mathematical Society, Providence, RI, 2005.

1 Department of Mathematics, Ankara University, 06100 TandoĞan, Ankara, TURKEY.

E-mail address: ekinugurlu@yahoo.com

2 Department of Mathematics, Suleyman Demirel University, 32260 Isparta, TURKEY.

E-mail address: bilenderpasaoglu@sdu.edu.tr 